\title{
Electron viscosity, current vortices and negative nonlocal resistance in graphene
}

\author{
Leonid Levitov ${ }^{1 \star}$ and Gregory Falkovich ${ }^{2,3 \star}$
}

\begin{abstract}
Quantum-critical strongly correlated electron systems are predicted to feature universal collision-dominated transport resembling that of viscous fluids ${ }^{1-4}$. However, investigation of these phenomena has been hampered by the lack of known macroscopic signatures of electron viscosity ${ }^{5-9}$. Here we identify vorticity as such a signature and link it with a readily verifiable striking macroscopic d.c. transport behaviour. Produced by the viscous flow, vorticity can drive electric current against an applied field, resulting in a negative nonlocal voltage. We argue that the latter may play the same role for the viscous regime as zero electrical resistance does for superconductivity. Besides offering a diagnostic that distinguishes viscous transport from ohmic currents, the sign-changing electrical response affords a robust tool for directly measuring the viscosity-to-resistivity ratio. A strongly interacting electron-hole plasma in high-mobility graphene $\mathrm{e}^{10-12}$ affords a unique link between quantum-critical electron transport and the wealth of fluid mechanics phenomena.
\end{abstract}

Symmetries and respective conservation laws play a central role in developing our understanding of strongly interacting states of matter. This is the case, in particular, for many systems of current interest, ranging from quantum-critical states in solids and ultracold atomic gases to quark-gluon plasmas ${ }^{1-4}$, which share common long-wavelength behaviour originating from the fundamental symmetries of spacetime. The ensuing energy and momentum conservation laws take the centre stage in these developments, defining hydrodynamics that reveals the universal collective behaviour. Powerful approaches based on conformal field theory and anti-de Sitter/conformal field theory (AdS/CFT) duality grant the well-established notions of fluid mechanics, such as viscosity and vorticity, an entirely new dimension ${ }^{13,14}$.

Despite their prominence and new paradigmatic role, viscous flows in strongly correlated systems have so far lacked directly verifiable macroscopic transport signatures. Surprisingly, this has been the case even for condensed matter systems where a wide variety of experimental techniques is available to probe collective behaviours. Identifying a signature that would do to viscous flows what zero electrical resistance did to superconductivity has remained an outstanding problem. The goal of this article is to point out that vorticity generated in viscous flows leads to a unique macroscopic transport behaviour that can serve as an unambiguous diagnostic of the viscous regime. Namely, we predict that vorticity of the shear flows generated by viscosity can result in a backflow of electrical current that can run against the applied field (see Fig. 1). The resulting negative nonlocal voltage therefore provides a clear signature of the collective viscous behaviour. Associated with it are characteristic sign-changing spatial patterns of electric potential (see Figs 1 and 2) which can be used to directly image vorticity and shear flows with modern scanning capacitance microscopy techniques ${ }^{15}$.

The negative electrical response, which is illustrated in Fig. 1, originates from the basic properties of shear flows. We recall that the collective behaviour of viscous systems results from momenta rapidly exchanged in carrier collisions while maintaining the net momentum conserved. Because momentum remains a conserved quantity collectively, it gives rise to a hydrodynamic momentum transport mode. Namely, momentum flows in space, diffusing transversely to the source-drain current flow and away from the nominal current path. A shear flow established as a result of this process generates vorticity and (for an incompressible fluid) a backflow in the direction reverse to the applied field. Such a complex and manifestly non-potential flow pattern has a direct impact on the electrical response, producing a reverse electric field acting opposite to the field driving the source-drain current (see Fig. 2). This results in a negative nonlocal resistance which persists even in the presence of fairly significant ohmic currents (see Fig. 2).

Attempts to connect electron theory with fluid mechanics have a long and interesting history, partially summarized in refs $8,16,17$. Early work on the viscosity of Fermi liquids made a connection with ultrasound damping ${ }^{18}$. Subsequently, Gurzhi introduced an electronic analogue of Poiseuille flow ${ }^{19}$. Related temperaturedependent phenomena in nonlinear transport were observed by deJong and Molenkamp ${ }^{16}$. Recent developments started with the theory of a hydrodynamic, collision-dominated quantum-critical regime advanced by Damle and Sachdev ${ }^{1}$. Andreev et al. argued that hydrodynamic contributions can dominate resistivity in systems with a large disorder correlation length ${ }^{7}$. Forcella et al. predicted that electron viscosity can impact electromagnetic field penetration in a striking way ${ }^{8}$. Davison et al. linked electron viscosity to linear resistivity of the normal state of the copper oxides ${ }^{20}$.

As a parallel development, recently there has been a surge of interest in the electron viscosity of graphene $e^{5,6,9,21,22}$. Quantumcritical behaviour is predicted to be particularly prominent in graphene ${ }^{10-12}$. Electron interactions in graphene are strengthened near charge neutrality owing to the lack of screening at low carrier densities $^{12,23}$. As a result, carrier collisions are expected to dominate transport in pristine graphene for a wide range of temperatures and dopings ${ }^{24}$. Furthermore, estimates of electronic viscosity near charge neutrality yield one of the lowest known viscosity-to-entropy ratios, which approaches the universal AdS/CFT bound ${ }^{5}$.

Despite the general agreement that graphene holds the key to electron viscosity, experimental progress has been hampered by the lack of easily discernible signatures in macroscopic transport. Several striking effects have been predicted, such as vortex shedding in the pre-turbulent regime induced by a strong current ${ }^{6}$, as well as nonstationary flow in a 'viscometer' comprised of an a.c.-driven

${ }^{1}$ Department of Physics, Massachusetts Institute of Technology, Cambridge, Massachusetts 02139, USA. ${ }^{2}$ Weizmann Institute of Science, Rehovot 76100, Israel. ${ }^{3}$ Institute for Information Transmission Problems, Moscow 127994, Russia. *e-mail: levitov@mit.edu; gregory.falkovich@weizmann.ac.il 


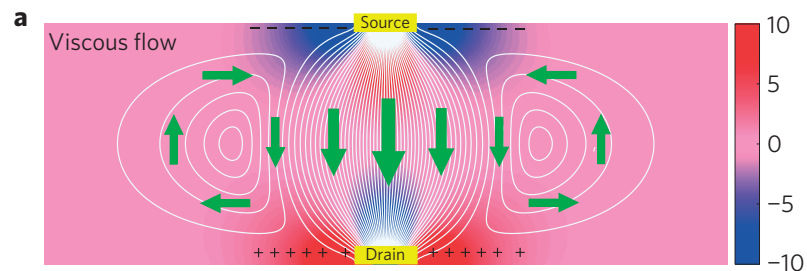

b

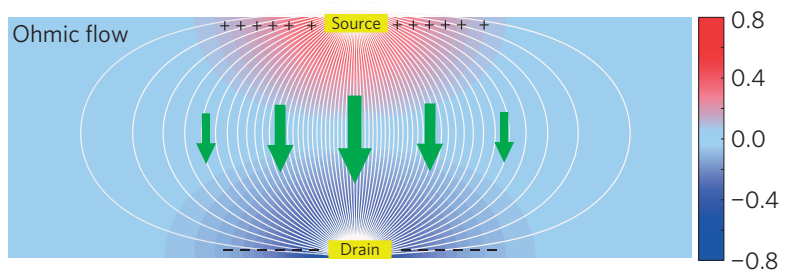

Figure 1 | Current streamlines and potential map for viscous and ohmic flows. White lines show current streamlines, colours show electrical potential, arrows show the direction of current. a, Mechanism of a negative electrical response: viscous shear flow generates vorticity and a backflow on the side of the main current path, which leads to charge buildup of the sign opposing the flow and results in a negative nonlocal voltage. Streamlines and electrical potential are obtained from equation (5) and equation (6). The resulting potential profile exhibits multiple sign changes and $\pm 45^{\circ}$ nodal lines, see equation (7). This provides directly measurable signatures of shear flows and vorticity. $\mathbf{b}$, In contrast, ohmic currents flow down the potential gradient, producing a nonlocal voltage in the flow direction.

Corbino disc ${ }^{9}$. These proposals, however, rely on fairly complex a.c. phenomena originating from high-frequency dynamics in the electron system. In each of these cases, as well as in those of refs 8,20 , a model-dependent analysis was required to delineate the effects of viscosity from 'extraneous' contributions. In contrast, the nonlocal d.c. response considered here is a direct manifestation of the collective momentum transport mode which underpins viscous flow, therefore providing an unambiguous, almost textbook, diagnostic of the viscous regime.

A nonlocal electrical response mediated by chargeless modes was found recently to be uniquely sensitive to quantities which are not directly accessible in electrical transport measurements, in particular spin currents and valley currents $\mathrm{s}^{25-27}$. In a similar manner, the nonlocal response discussed here gives a diagnostic of viscous transport, which is more direct and powerful than any approaches based on local transport.

There are several aspects of the electron system in graphene that are particularly well suited for studying electronic viscosity. First, the momentum-nonconserving Umklapp processes are forbidden in two-body collisions because of graphene's crystal structure and symmetry. This ensures the prominence of momentum conservation and associated collective transport. Second, although carrier scattering is weak away from charge neutrality, it can be enhanced by several orders of magnitude by tuning the carrier density to the neutrality point. This allows one to cover the regimes of high and low viscosity, respectively, in a single sample. Last, the two-dimensional structure and atomic thickness makes the electronic states in graphene fully exposed and amenable to sensitive electric probes.

To show that the timescales are favourable for the hydrodynamical regime, we will use parameter values estimated for pristine graphene samples which are almost defect free, such as freestanding graphene ${ }^{28}$. Kinematic viscosity can be estimated as the momentum diffusion coefficient $v \approx(1 / 2) v_{\mathrm{F}}^{2} \gamma_{\mathrm{ee}}^{-1}$, where $\gamma_{\mathrm{ee}}$ is the carrier-carrier scattering rate, and $\nu_{\mathrm{F}}=10^{6} \mathrm{~m} \mathrm{~s}^{-1}$ for graphene. According to Fermi-liquid theory, this rate behaves as $\gamma_{\mathrm{ee}} \sim\left(k_{\mathrm{B}} T\right)^{2} / E_{\mathrm{F}}$ in the degenerate limit (that is, away from charge neutrality), which leads to large $v$ values. Near charge neutrality,

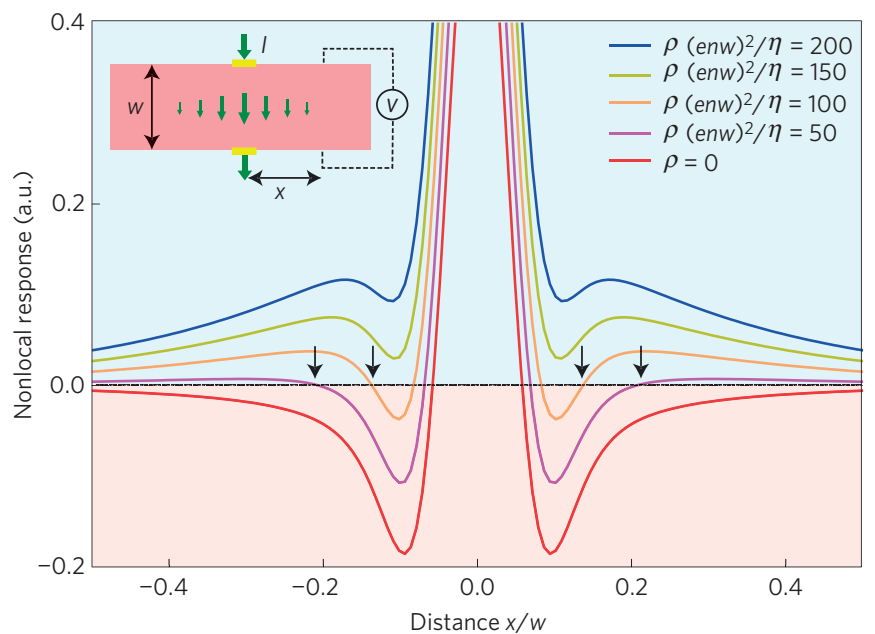

Figure $\mathbf{2}$ | Nonlocal response for different resistivity-to-viscosity ratios $\rho / \eta$. Voltage $V(x)$ is plotted at a distance $x$ from current leads obtained from equation (12) for the set-up shown in the inset. The voltage is positive in the ohmic-dominated region at large $|x|$ and negative in the viscosity-dominated region closer to the leads (positive values at even smaller $|x|$ reflect the finite contact size $a \approx 0.05 \mathrm{w}$ used in simulation). Viscous flow dominates up to fairly large resistivity values, resulting in the negative response persisting up to values as large as $\rho(\text { enw })^{2} / \eta \approx 120$. Positive and negative voltage regions are marked by blue and pink, respectively. Nodal points, marked by arrows, are sensitive to the $\rho / \eta$ value, which provides a way to directly measure viscosity (see text).

however, the rate $\gamma_{\mathrm{ee}}$ grows and $v$ approaches the AdS/CFT limitnamely $s \hbar / 4 \pi k_{\mathrm{B}}$, where $s$ is entropy density. Refs 12,23 estimate this rate as $\gamma_{\mathrm{ee}} \approx A \alpha^{2} k_{\mathrm{B}} T / \hbar$, where $\alpha$ is the interaction strength. For $T=100 \mathrm{~K}$, assuming $E_{\mathrm{F}}=0$ and approximating the prefactor as $A \approx 1$ (refs 12,23), this predicts characteristic times as short as $\gamma_{\mathrm{ee}}^{-1} \approx 80 \mathrm{fs}$. Disorder scattering can be estimated from the measured mean free path values, which reach a few microns at large doping ${ }^{29}$. Using the momentum relaxation rate square-root dependence on doping, $\gamma_{\mathrm{p}} \propto n^{-1 / 2}$, and estimating it near charge neutrality, $n \lesssim 10^{10} \mathrm{~cm}^{-2}$, gives times $\gamma_{\mathrm{p}}^{-1} \sim 0.5 \mathrm{ps}$ which are longer than the values $\gamma_{\mathrm{ee}}^{-1}$ estimated above. The inequality $\gamma_{\mathrm{p}} \ll \gamma_{\mathrm{ee}}$ justifies our hydrodynamical description of transport.

Momentum transport in the hydrodynamic regime is described by the continuity equation for momentum density,

$$
\partial_{t} p_{i}+\partial_{j} T_{i j}=-\gamma_{\mathrm{p}} p_{i}, \quad T_{i j}=P \delta_{i j}+\mu v_{i} v_{j}+T_{i j}^{(\mathrm{v})}
$$

where $T_{i j}$ is the momentum flux tensor, $P$ and $\mu$ are pressure and mass density, and $\mathbf{v}$ is the carrier drift velocity. The quantity $\gamma_{\mathrm{p}}$, introduced above, describes electron-lattice momentum relaxation due to disorder or phonons, which we will assume to be small compared to the carrier scattering rate. We can relate pressure to the electrochemical potential $\Phi$ through $P=e \int_{n_{0}}^{n} \Phi\left(n^{\prime}\right) \mathrm{d} n^{\prime}$. Here we work at degeneracy, $E_{\mathrm{F}} \gg k_{\mathrm{B}} T$, ignoring the entropic/thermal contributions, and approximating $P \approx e\left(n-n_{0}\right) \Phi$, with $n$ the particle number density. While carrier scattering is suppressed at degeneracy as compared to its value at $E_{\mathrm{F}}=0$, here we assume that the carrier-carrier scattering remains faster than the disorder scattering, as required for the validity of hydrodynamics. Viscosity contributes to the momentum flux tensor through

$$
T_{i j}^{(\mathrm{v})}=\eta\left(\partial_{i} v_{j}+\partial_{j} v_{i}\right)+(\zeta-\eta) \partial_{k} v_{k} \delta_{i j}
$$

where $\eta$ and $\zeta$ are the first and second viscosity coefficients. For drift velocities smaller than plasmonic velocities, transport in charged systems is described by an incompressible flow with a 
divergenceless velocity field, $\partial_{i} v_{i}=0$. In this work, we consider the limit of low Reynolds number, $\mu v_{i} v_{j} \ll \eta\left(\partial_{i} v_{j}+\partial_{j} v_{i}\right)$, such that the role of viscosity is most prominent. At linear order in $\mathbf{v}$, we obtain an electronic Navier-Stokes equation

$$
\partial_{t} p_{i}-\eta \nabla^{2} v_{i}+\gamma_{\mathrm{p}} p_{i}=-\partial_{i} P
$$

This equation describes momentum transport: imparted by the external field $\mathbf{f}=-\nabla P$, momentum flows to the system boundary where dissipation takes place. It is therefore important to endow equation (3) with suitable boundary conditions. In fluid mechanics this is described by the no-slip boundary condition $\mathbf{v}=0$. We use a slightly more general boundary condition

$$
v_{\perp}=0, \quad v_{\|}=-\alpha \partial_{\|} P
$$

where the subscripts $\perp$ and $\|$ indicate the velocity and derivative components normal and tangential to the boundary. The second relation in equation (4) generalizes the no-slip condition to account for non-hydrodynamical effects in the boundary layer on the scales $\gtrsim l=v / \gamma_{\text {ee }}$. The model (4), equipped with the parameter $\alpha$, provides a convenient way to assess the robustness of our predictions.

It is instructive to consider a current flowing down a long strip of a finite width. A steady viscous flow features a non-uniform profile in the strip cross-section governed by the momentum flow to the boundary. Equation (3), applied to a strip $0<y<w$, yields $\left(-\eta \partial_{y}^{2}+\gamma_{\mathrm{p}} m n\right) v(y)=e n E$, where $v(y)$ and $E$ are the drift velocity and electric field directed along the strip (and $m$ is an effective mass defined through the relation $\mathbf{p}=m n \mathbf{v})$. Setting $\alpha$ and $\gamma_{\mathrm{p}}$ to zero for simplicity, we find a parabolic profile $v(y)=A y(w-y)$, where $A=n e E / 2 \eta$ and $\eta=m n v$. The nonzero shear $\partial_{y} v=A(w-2 y)$ describes momentum flow to the boundary. The net current $I=\int_{0}^{w} n e v\left(y^{\prime}\right) \mathrm{d} y^{\prime}=\left(n^{2} e^{2} / 12 \eta\right) w^{3} E$, scaling as a cube of the strip width, is the electronic analogue of the Poiseuille law. Being distinct from the linear scaling $I \propto w E$ in the ohmic regime, the cubic scaling can in principle be used to identify the viscous regime. It is interesting to put the current-field relation in a 'Drude' form using kinematic viscosity: $I=\left(n e^{2} \tau_{w} / m\right) w E$, with $\tau_{w}=w^{2} / 12 v$ being an effective scattering time. Evaluating the latter as $\tau_{w} \approx(1 / 6)\left(w / v_{\mathrm{F}}\right)^{2} \gamma_{\mathrm{ee}}$, we find values that, for realistic system parameters, can greatly exceed the naive estimate $\gamma_{\mathrm{p}}^{-1}=w / \nu_{\mathrm{F}}$ based on the ballistic transport picture. This remarkable observation was first made by Gurzhi, see ref. 19 .

Next, we proceed to analyse the nonlocal response in a strip with transverse current injected and drained through a pair of contacts, as pictured in Fig. 1. Unlike the above case of longitudinal current, here the potential profile is not set externally but must be obtained from equation (3). The analysis is facilitated by introducing a stream function via $\mathbf{v}=\mathbf{z} \times \nabla \psi$, which solves the incompressibility condition. At first we will completely ignore the ohmic effects, setting $\alpha$ and $\gamma_{p}$ to zero as above, which leads to a biharmonic equation $\left(\partial_{x}^{2}+\partial_{y}^{2}\right)^{2} \psi=0$ with the boundary conditions $v_{x}=0$, $n e v_{y}=I \delta(x)$ for $y=0, w$. Using a Fourier transform in $x$, we write $\psi(x, y)=(2 \pi)^{-1} \int \mathrm{d} k \mathrm{e}^{i k x} \psi_{k}(y)$ and then determine $\psi_{k}(y)$ separately for each $k$ (see Supplementary Information). Inverting the Fourier transform gives the stream function

$$
\begin{aligned}
\psi(x, y)= & \frac{I}{n e} \int \frac{\mathrm{d} k \mathrm{e}^{i k x}}{2 \pi i k}\left[\frac{\mathrm{e}^{k y}+\mathrm{e}^{k(w-y)}}{\mathrm{e}^{k w}+1}\right. \\
& \left.+a_{k}[y \sinh k(w-y)+(w-y) \sinh k y]\right]
\end{aligned}
$$

where we defined $a_{k}=k \tanh (k w / 2) /(k w+\sinh k w)$. Contours (isolines) of $\psi$ give the streamlines for the flow shown in Fig. 1. Although most of them are open lines connecting source and drain, some streamlines form loops. The latter define vortices occurring on both sides of the current path. Numerically, we find that vortex centres are positioned very close to $x= \pm w$ (see Supplementary Information).

We can now explore the electrical potential of the viscous flow. The latter can be found directly from $\psi(x, y)$, giving

$$
\phi(x, y)=\frac{\beta I}{2} \int \mathrm{d} k \mathrm{e}^{i k x} a_{k}[\sinh k(y-w)+\sinh k y]
$$

where we defined $\beta=2 \eta /\left(\pi n^{2} e^{2}\right)$ (see Supplementary Information). As illustrated in Fig. 1, equation (6) predicts a peculiar sign-changing spatial dependence, with two pairs of nodal lines crossing at contacts. To understand this behaviour, we evaluate $\phi(x, y)$ explicitly in the regions near contacts $(x, y)=(0,0),(0, w)$. Near the first contact, approximating $\tanh (k w / 2) \approx \operatorname{sgn} k, \sinh k y \approx(1 / 2) \mathrm{e}^{|k| y} \operatorname{sgn} k$, and so on, we find

$$
\phi(x, y) \approx \frac{\beta I}{2} \int \mathrm{d} k \mathrm{e}^{i k x}|k| \mathrm{e}^{-|k| y}=\frac{\beta I\left(y^{2}-x^{2}\right)}{\left(y^{2}+x^{2}\right)^{2}}
$$

$(|x|,|y| \ll w)$. Equation (7) predicts an inverse-square dependence versus distance from contacts and also the presence of two nodal lines running at $\pm 45^{\circ}$ angles relative to the nominal current path. Similar behaviour is found near the other contact, $\phi(x, y) \approx-\left(\beta\left((w-y)^{2}-x^{2}\right) /\left((w-y)^{2}+x^{2}\right)^{2}\right) I$. We note that the $r^{-2}$ power law dependence is much stronger than the $\ln r$ dependence expected in the ohmic regime. This, as well as multiple sign changes, provides a clear signature of a viscous flow.

The nonlocal voltage measured at a finite distance from the current leads (see schematic in Fig. 2 inset) can be evaluated as $V(x)=\phi(x, w)-\phi(x, 0)$. From equation (7) we predict a voltage that is falling off as $x^{-2}$ and is of a negative sign:

$$
V(x) \approx-\frac{2 \beta}{x^{2}} I
$$

$(|x| \lesssim w)$. Microscopically, negative voltage originates from a viscous shear flow which creates vorticity and backflow on both sides of the current path (see Fig. 1).

Numerically, we see that the negative response persists to arbitrarily large distances, see the $\rho=0$ curve in Fig. 2. The sign change at very short $x$, evident in Fig. 2 , arises owing to a finite contact size. We model it by replacing the delta function in the boundary condition for the current source by a Lorentzian, nev $=I a / \pi\left(x^{2}+a^{2}\right)$ at $y=0, w$. After making appropriate changes in the above derivation (namely, plugging $\mathrm{e}^{-a|k|}$ under the integral) we find

$$
V(x) \approx-\frac{\beta I}{(x-i a)^{2}}+\text { c.c. }=-\frac{2 \beta I\left(x^{2}-a^{2}\right)}{\left(x^{2}+a^{2}\right)^{2}}
$$

This expression exhibits a sign change at $x=a$ (representing 'the contact edge') and is negative for all $|x|>a$, that is, everywhere outside contacts (this is further discussed in Supplementary Information).

It is interesting to probe to what extent the negative response is sensitive to boundary conditions-in particular to the noslip assumption. Extending the above analysis to the boundary conditions with nonzero $\alpha$ in equation (4), we find the nonlocal response of the form

$$
V(x)=\beta I \int \mathrm{d} k \mathrm{e}^{i k x} \frac{k \tanh (k w / 2) \sinh k w}{k w+\left(1+\tilde{\alpha} k^{2}\right) \sinh k w}
$$

where $\tilde{\alpha}=2 \eta \alpha / n e$. The expression under the integral represents an even function of $k$ with a zero at $k=0$ and a symmetric 

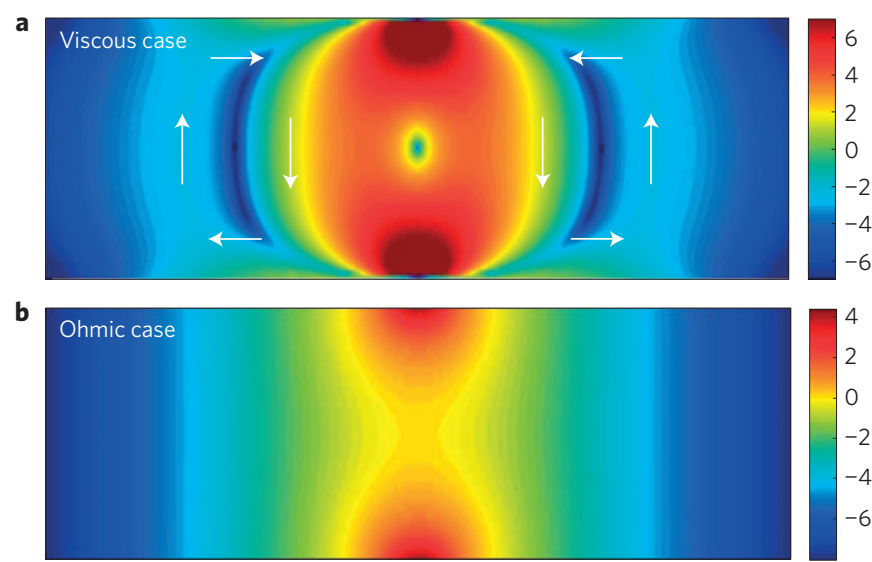

Figure 3 | Heating patterns for viscous and ohmic flows. a, Viscous flow results in a highly complex heating pattern with intense hot spots near contacts and cold arc-shaped patches at vortices surrounded by warmer regions. (Also note a cold spot at the centre where the flow is locally uniform and thus $W=0$, see equation (13).) White arrows show current direction. $\mathbf{b}$, Ohmic flow shows an essentially featureless heat production rate decaying monotonously away from contacts.

double-peak structure. The peaks roll off at $|k| \sim \tilde{\alpha}^{-1 / 2}$, which sets an ultraviolet cutoff for the integral similar to that above for a finitesize contact model. Our numerical analysis shows that this is indeed the case, with a finite $\alpha$ translating into an effective contact size $a \approx \tilde{\alpha}^{1 / 2}$. In other words, the modified boundary conditions can alter the response in the proximity of the contact while rendering it unaffected at larger distances.

So far we have ignored the bulk momentum relaxation, setting $\gamma_{\mathrm{p}}=0$ in equation (3). We now proceed to show that the signatures of viscous flow identified above are robust in the presence of a background ohmic resistivity $\rho=m \gamma_{\mathrm{p}} / n e^{2}$ so long as it is not too strong. The dimensionless parameter which governs the respective roles of resistivity versus viscosity is

$$
\epsilon=\rho(e n w)^{2} / \eta \approx 2 \gamma_{\mathrm{ee}} \gamma_{\mathrm{p}}\left(w / \nu_{\mathrm{F}}\right)^{2}
$$

For the values $\gamma_{\mathrm{ee}}$ and $\gamma_{\mathrm{p}}$ quoted above, and taking $w=1 \mu \mathrm{m}$, one obtains $\epsilon \sim 10$. Incorporating finite resistivity in the calculation is uneventful, yielding a response

$$
V(x)=I \int \frac{\mathrm{d} k}{\pi k} \frac{\rho \mathrm{e}^{i k x}\left(\mathrm{e}^{q w}-1\right)\left(\mathrm{e}^{k w}-1\right) q}{q_{+}\left(\mathrm{e}^{q w}-\mathrm{e}^{k w}\right)+q_{-}\left(\mathrm{e}^{q^{w}} \mathrm{e}^{k w}-1\right)}
$$

where $q^{2}=k^{2}+\epsilon w^{-2}, q_{ \pm}=q \pm k$ (see Supplementary Information). For $\epsilon=0$ we recover the pure viscous result, which is negative, whereas for $\epsilon \rightarrow \infty$ equation (12) gives the well-known ohmic result $V(x)=(\rho / \pi) \ln [\operatorname{coth}(\pi x / 2 w)]$, which is positive. With both $\eta$ and $\rho$ nonzero, the resistance given by equation (12) is positive at large $x$ but remains negative close to the contact. The dimensionless threshold that determines the possibility of negative electric response depends on the actual contact size. For the values used in Fig. 2 the negative response occurs even at fairly high resistivity values corresponding to $\epsilon \lesssim 100$.

The robustness of the negative response can be understood by noting that viscosity is the coefficient of the highest derivative term in equation (3) and thus dominates at short distances $x \lesssim x_{*}=\sqrt{\eta / \rho(e n)^{2}}$. The pervasive character of the negative response, manifest in Fig. 2, will facilitate experimental detection of viscous transport. The positions of the nodes, marked by arrows in Fig. 2, vary with the ratio $\rho / \eta$, which provides a convenient way to directly measure the electronic viscosity.
The hydrodynamic transport regime also features interesting thermal effects. At leading order in the flow velocity those are dominated by convective heat transfer, described by a proportionality relation between entropy flux and flow velocity $v$ (to be discussed elsewhere). At higher order in $v$, besides the conventional Joule heating, vorticity of a viscous flow manifests itself in heat production

$$
W=\eta\left(\partial_{i} v_{j}+\partial_{j} v_{i}\right)^{2}=2 \eta\left|\left(\partial_{x}+i \partial_{y}\right)^{2} \psi(x, y)\right|^{2}
$$

The vorticity-induced heating pattern, shown in Fig. 3, features hot spots near contacts and cold arc-shaped elongated patches in vorticity regions. In contrast, for an ohmic flow the pattern is essentially featureless. This makes viscous flows an interesting system to explore with the nanoscale temperature scanning techniques ${ }^{30}$.

We finally note that there are tantalizing parallels-both conceptual and quantitative-between electronic viscous flows and current microfluidics systems. Namely, a model essentially identical to equation (3) describes the low-Reynolds (microfluidic) flow between two plates separated by a distance $h$, where $\gamma_{\mathrm{p}} m n=$ $12 \eta / h^{2}$ (it also describes viscous electron flow in a 3D slab, see Supplementary Information). A new research area at the frontier of nanoscience and fluid mechanics, microfluidics aims to manipulate and control fluids at a nanoscale with the ultimate goal of developing new lab-on-a-chip microtechnologies. Graphene, which can be easily patterned into any shapes without compromising its excellent qualities, can become a basis of electronic microfluidics, with multiple applications in information processing and nanoscale charge and energy transport that remain to be explored.

Note added in proof: After submitting our work, it came to our attention that negative resistance has recently been observed in graphene (see refs 31,32 ).

Received 31 July 2015; accepted 15 January 2016; published online 22 February 2016

\section{References}

1. Damle, K. \& Sachdev, S. Nonzero-temperature transport near quantum critical points. Phys. Rev. B 56, 8714-8733 (1997).

2. Kovtun, P. K., Son, D. T. \& Starinets, A. O. Viscosity in strongly interacting quantum field theories from black hole physics. Phys. Rev. Lett. 94, 111601 (2005).

3. Son, D. T. Vanishing bulk viscosities and conformal invariance of the unitary Fermi gas. Phys. Rev. Lett. 98, 020604 (2007).

4. Karsch, K., Kharzeev, D. \& Tuchin, K. Universal properties of bulk viscosity near the QCD phase transition. Phys. Lett. B 663, 217-221 (2008).

5. Müller, M., Schmalian, J. \& Fritz, L. Graphene: a nearly perfect fluid. Phys. Rev. Lett. 103, 025301 (2009).

6. Mendoza, M., Herrmann, H. J. \& Succi, S. Preturbulent regimes in graphene flow. Phys. Rev. Lett. 106, 156601 (2011).

7. Andreev, A. V., Kivelson, S. A. \& Spivak, B. Hydrodynamic description of transport in strongly correlated electron systems. Phys. Rev. Lett. 106, 256804 (2011).

8. Forcella, D., Zaanen, J., Valentinis, D. \& van der Marel, D. Electromagnetic properties of viscous charged fluids. Phys. Rev. B 90, 035143 (2014).

9. Tomadin, A., Vignale, G. \& Polini, M. Corbino disk viscometer for 2D quantum electron liquids. Phys. Rev. Lett. 113, 235901 (2014).

10. González, J., Guinea, F. \& Vozmediano, M. A. H. Non-Fermi liquid behavior of electrons in the half-filled honeycomb lattice (A renormalization group approach). Nucl. Phys. B 424, 595-618 (1994).

11. Sheehy, D. E. \& Schmalian, J. Quantum critical scaling in graphene. Phys. Rev. Lett. 99, 226803 (2007).

12. Fritz, L., Schmalian, J., Müller, M. \& Sachdev, S. Quantum critical transport in clean graphene. Phys. Rev. B 78, 085416 (2008).

13. Son, D. T. \& Starinets, A. O. Viscosity, black holes, and quantum field theory. Annu. Rev. Nucl. Part. Sci. 57, 95118 (2007)

14. Sachdev, S. \& Muller, M. Quantum criticality and black holes. J. Phys. Condens. Matter 21, 164216 (2009). 
15. Yoo, M. J. et al. Scanning single-electron transistor microscopy: imaging individual charges. Science 276, 579-582 (1997).

16. de Jong, M. J. M. \& Molenkamp, L. W. Hydrodynamic electron flow in high-mobility wires. Phys. Rev. B 51, 13389-13402 (1995).

17. Jaggi, R. Electron-fluid model for the dc size effect. J. Appl. Phys. 69, 816-820 (1991)

18. Lifshitz, E. M. \& Pitaevskii, L. P. Physical Kinetics (Pergamon Press, 1981).

19. Gurzhi, R. N. Hydrodynamic effects in solids at low temperature. Sov. Phys. Usp. 11, 255 (1968); http://go.nature.com/Lg1Zp1

20. Davison, R. A., Schalm, K. \& Zaanen, J. Holographic duality and the resistivity of strange metals. Phys. Rev. B 89, 245116 (2014).

21. Principi, A., Vignale, G., Carrega, M. \& Polini, M. Bulk and shear viscosities of the $2 \mathrm{D}$ electron liquid in a doped graphene sheet. Preprint at http://arXiv.org/abs/1506.06030 (2015).

22. Cortijo, A., Ferreirós, Y., Landsteiner, K. \& Vozmediano, M. A. H. Hall viscosity from elastic gauge fields in Dirac crystals. Phys. Rev. Lett. 115, 177202 (2015).

23. Kashuba, A. B. Conductivity of defectless graphene. Phys. Rev. B 78, 085415 (2008).

24. Narozhny, B. N., Gornyi, I. V., Titov, M., Schütt, M. \& Mirlin, A. D. Hydrodynamics in graphene: Linear-response transport. Phys. Rev. B 91, 035414 (2015).

25. Abanin, D. A., Gorbachev, R. V., Novoselov, K. S., Geim, A. K. \& Levitov, L. S. Giant spin-Hall effect induced by the Zeeman interaction in graphene. Phys. Rev. Lett. 107, 096601 (2011).

26. Abanin, D. A. et al. Giant nonlocality near the Dirac point in graphene. Science 332, 328330 (2011)

27. Gorbachev, R. V. et al. Detecting topological currents in graphene superlattices. Science 346, 448-451 (2014).

28. Bolotin, K. I. et al. Ultrahigh electron mobility in suspended graphene. Solid State Commun. 146, 351-355 (2008).
29. Taychatanapat, T., Watanabe, K., Taniguchi, T. \& Jarillo-Herrero, P. Electrically tunable transverse magnetic focusing in graphene. Nature Phys. $\mathbf{9}$, 225-229 (2013)

30. Kucsko, G. et al. Nanometre-scale thermometry in a living cell. Nature 500, 54-58 (2013).

31. Bandurin, D. A. et al. Negative local resistance due to viscous electron backflow in graphene. Preprint at http://arxiv.org/abs/1509.04165 (2015).

32. Torre, I., Tomadin, A., Geim, A. K. \& Polini, M. Nonlocal transport and the hydrodynamic shear viscosity in graphene. Phys. Rev. B 92, 165433 (2015).

\section{Acknowledgements}

We acknowledge support of the Center for Integrated Quantum Materials (CIQM) under NSF award 1231319 (L.L.), partial support by the US Army Research Laboratory and the US Army Research Office through the Institute for Soldier Nanotechnologies, under contract number W911NF-13-D-0001 (L.L.), MISTI MIT-Israel Seed Fund (L.L. and G.F.), the Israeli Science Foundation (grant 882) (G.F.) and the Russian Science Foundation (project 14-22-00259) (G.F.).

\section{Author contributions}

All authors contributed to all aspects of this work

\section{Additional information}

Supplementary information is available in the online version of the paper. Reprints and permissions information is available online at www.nature.com/reprints.

Correspondence and requests for materials should be addressed to L.L. or G.F.

\section{Competing financial interests}

The authors declare no competing financial interests. 